\title{
Mediation effect of herpes zoster derived by statin use on cardiovascular disease risk
}

\author{
Sung-Han Kim ${ }^{1,}$, Sung-Cheol Yun ${ }^{2,}$, Young-Ho Khang ${ }^{3}$, Min-Chul Kim ${ }^{4}$, Sun Uck Kwon ${ }^{5}$, Gyung-Min Park ${ }^{6}$, \\ Young-Rak Cho ${ }^{7}$, Kwang Min Lee ${ }^{7}$, and Moo Hyun Kim
}

\author{
Departments of ${ }^{1}$ Infectious \\ Diseases, ${ }^{2}$ Clinical Epidemiology \\ and Biostatistics, Asan Medical \\ Center, University of Ulsan College \\ of Medicine, Seoul; ' ${ }^{3}$ Department \\ of Health Policy and Management, \\ Seoul National University College \\ of Medicine, Seoul; ${ }^{4}$ Division of \\ Infectious Diseases, Department \\ of Internal Medicine, Chung- \\ Ang University Hospital, Seoul; \\ ${ }^{5}$ Department of Neurology, Asan \\ Medical Center, University of \\ Ulsan College of Medicine, Seoul; \\ ${ }^{6}$ Department of Cardiology, \\ Ulsan University Hospital, Ulsan; \\ ${ }^{7}$ Department of Cardiology, Dong-A \\ University College of Medicine, \\ Busan, Korea
}

Received: January 12, 2019 Revised : February 18, 2019 Accepted: March 7, 2019

\section{Correspondence to}

Moo Hyun Kim, M.D.

Department of Cardiology, Dong-A University College of

Medicine, 26 Daesingongwon-ro, Seo-gu, Busan 49201, Korea

Tel: +82-51-240-2976

Fax: +82-51-255-2177

E-mail:kimmh@dau.ac.kr

https://orcid.org/0000-0003-

3468-6453

*'These authors contributed equally to this work.
Background/Aims: Although statins are widely used to reduce the risk of cardiovascular disease (CVD) including stroke and myocardial infarction (MI), it is reported that statin use increases the incidence of herpes zoster (HZ) that is associated with increased risk of CVD. So, we evaluated the mediation effect of $\mathrm{HZ}$ caused by statin use on CVD.

Methods: We analyzed a prospective cohort from the National Health Insurance Service-database of South Korea. All individuals received a medical check-up and were followed-up from 2002 to 2013.

Results: A total of 275,382 individuals $>40$ years old were followed up for 11 years from 2003. Of these, 11,415 people (4\%) were classified as statin users and 263,967 $(96 \%)$ as non-statin users. Those who used statins had significantly lower risks of cardiovascular events, stroke, and MI compared with non-statin users; the adjusted hazard ratios in the multivariate analysis were 0.90 ( $95 \%$ confidence interval [CI], 0.82 to 0.98 ), 0.88 (95\% CI, 0.80 to 0.98 ), and 0.91 (95\% CI, 0.79 to 1.07), respectively. When we calculated the mediating effect of cardiovascular events by statin use through HZ, $11.6 \%$ of the total beneficial effect of cardiovascular events by statin use was mitigated through the occurrence of $\mathrm{HZ}$ caused by statin use. This mediating effect was higher in the younger age group (<60 years).

Conclusions: This study showed that statin use reduced CVD by $10 \%$, but the protective effect of statin use against CVD was mitigated by approximately $10 \%$ through the development of HZ caused by statin use.

Keywords: Herpes zoster; Stroke; Myocardial infarction

\section{INTRODUCTION}

Herpes zoster (HZ), also known as shingles, occurs in about $20 \%$ or more of the population, mainly in the el- derly [1]. Previous epidemiologic studies revealed that $\mathrm{HZ}$ increases the risk of cardiovascular events, including stroke and myocardial infarction (MI) ([b] in Supplementary Fig. 1) [1-5]. It is also well known that statin use 
reduces the risk of stroke and MI ([c] in Supplementary Fig. 1) [6]; however, recent studies have consistently reported that statin use increases the incidence of $\mathrm{HZ}$ ([a] in Supplementary Fig. 1) [7-9]. Therefore, we can expect that the protective effect of statin use against stroke and MI may be mitigated through the development of HZ, and that this unwanted effect would be counterbalanced through zoster vaccination in statin users with high risk of stroke and MI. The aim of this study is to measure the mediation effect of HZ caused by statin use to prevent stroke and MI.

\section{METHODS}

\section{Database and study population}

All individuals in South Korea are covered by the single-payer Korea National Health Insurance Service (NHIS). The Korea National Health Insurance has been operated based on a fee-for-service payment system for both inpatient and outpatient services and thus all of health care utilization data including medication history are available. The Korea NHIS built up the national representative cohort database, which was comprised of 1,025,340 nationally representative random subjects in 2002 , amounting to $2 \%$ of the entire population [10]. Proportionate stratified random sampling was used based on a total of 1,476 strata (two categories for sex, 18 categories for age group, and 41 categories for income). This cohort was followed-up over 12 years until 2013, and random samples of new infants were added to compensate for the loss of individuals due to death or emigration for each year between 2003 and 2013. The data consist of complete medical records, including socio-demographic variables, diagnostic codes, deaths, medical service use, and health examinations. Recently, the NHIS publicly released this National Health Insurance Service-National Sample Cohort 2002-2013 (NHISNSC 2002-2013). Detailed information on the NHIS-NSC profile was described in a previous study [10]. We used a subset database of the NHIS-NSC, which consisted of approximately 570,000 people who received a medical check-up without charge that provided comprehensive social and medical information about the population. The protocol for this study was approved by the Institutional Review Board of Asan Medical Center(S2015-0982) and Dong-A University Hospital (DAUHIRB-17-134), informed consent was waived by the board.

\section{Identification of herpes zoster, stroke, AMI, and other confounding factors}

We identified patients who were diagnosed with HZ, stroke, and MI by use of the relevant diagnostic codes of the International Classification of Diseases, Tenth Revision (ICD-10). The cases of HZ were comprised of VZV meningitis (Bo20), VZV encephalitis (Bo21), zoster in other divisions of the trigeminal nerve (Bo22), zoster ophthalmicus (Bo23), and other types of zoster (Bo27, Bo28, or Bo29). The cardiovascular disease (CVD) referred to all episodes of stroke and MI. Stroke consisted of ischemic brain infarction (I63), hemorrhage stroke (I60, I61, or I62), and unspecified stroke (I64). MI included myocardial infarction (I21, I22) and complications following myocardial infarction (I23).

In addition, clinical characteristics that could potentially become confounding factors for HZ, stroke, and MI were searched using ICD-10 code or medical records; these included age, gender, body mass index, overweight, cigarette smoking, alcohol consumption, physical activity, income, hypertension, diabetes (fasting plasma glucose), dyslipidemia (total cholesterol), angina pectoris, transient ischemic attack, heart failure, atrial fibrillation/ flutter, valvular heart disease, chronic renal disease, peripheral vascular disease, chronic liver disease, rheumatoid disease, inflammatory bowel disease, malignancy, transplantation recipients of solid organs or hematopoietic stem cell, and human immunodeficiency virus infection. We considered the variables listed in Table 1 as potential confounders between statin use and CVD, including stroke and MI, between statin use and HZ, and between HZ and CVD, and we adjusted for these variables in the main analysis. These covariates were selected a priori as common risk factors of HZ and CVD (Supplementary Fig. 1). Chronic pulmonary disease is a major risk factor for HZ and CVD [11]. As such, we did not include chronic pulmonary disease as a variable in the main analysis to quantify the impact of unmeasured mediator-outcome confounding on mediation effect.

\section{Study design and outcomes}

Initially, 569,999 individuals were enrolled in this cohort (Fig. 1). Of this population, 148 persons under 18 
Table 1. Univariate associations of baseline variables between statin users and non-statin users

\begin{tabular}{|c|c|c|c|c|}
\hline Characteristic & $\begin{array}{c}\text { Total } \\
(\mathrm{n}=275,382)\end{array}$ & $\begin{array}{l}\text { Non-statin users } \\
(\mathrm{n}=263,967)\end{array}$ & $\begin{array}{l}\text { Statin users } \\
(\mathrm{n}=11,415)\end{array}$ & $p$ value \\
\hline Age, yr & $54.0 \pm 10.2$ & $53.8 \pm 10.2$ & $59.9 \pm 9.3$ & $<0.001$ \\
\hline $41-50$ & $128,587(47)$ & $126,505(48)$ & $2,082(18)$ & $<0.001$ \\
\hline $51-60$ & $77,309(28)$ & $73,185(28)$ & $4,214(36)$ & \\
\hline $61-70$ & $48,428(18)$ & $44,715(17)$ & $3,713(33)$ & \\
\hline$\geq 71$ & $21,058(8)$ & $19,562(7)$ & $1,496(13)$ & \\
\hline Male sex & $130,645(47)$ & $126,352(48)$ & $4,293(38)$ & $<0.001$ \\
\hline BMI, kg/m² & $23.9 \pm 3.1$ & $23.9 \pm 3.1$ & $25 \cdot 4 \pm 3.3$ & $<0.001$ \\
\hline Obesity, BMI $\geq 25 \mathrm{~kg} / \mathrm{m}^{2}$ & $94,149(34)$ & $88,226(33)$ & $5,923(52)$ & $<0.001$ \\
\hline Smoking & & & & $<0.001$ \\
\hline Non-smoker & $180,453(66)$ & $172,293(65)$ & $8,160(72)$ & \\
\hline Ex-smoker & $16,147(6)$ & $15,055(6)$ & $1,092(10)$ & \\
\hline Current smoker & $59,513(22)$ & $57,848(22)$ & $1,665(15)$ & \\
\hline Unknown & $19,269(7)$ & $18,771(7)$ & $498(4)$ & \\
\hline Drinking & & & & $<0.001$ \\
\hline Non-drinker & $160,165(58)$ & $152,174(58)$ & 7,991 (70) & \\
\hline$\leq$ Twice per week & $75,073(27)$ & $72,956(28)$ & $2,117(19)$ & \\
\hline$\geq 3$ times per week & $34,772(13)$ & $33,685(13)$ & $1,087(10)$ & \\
\hline Unknown & $5,327(2)$ & $5,152(2)$ & $220(2)$ & \\
\hline Exercise & & & & $<0.001$ \\
\hline None & $158,404(58)$ & $152,056(58)$ & $6,348(56)$ & \\
\hline$\leq$ Twice per week & $60,589(22)$ & $58,257(22)$ & $2,332(20)$ & \\
\hline$\geq 3$ times per week & $50,267(18)$ & $47,769(18)$ & $2,498(22)$ & \\
\hline Unknown & $6,122(2)$ & $5,885(2)$ & $237(2)$ & \\
\hline Economic class & & & & $<0.001$ \\
\hline $\mathrm{I}$ & $48,354(18)$ & $46,277(18)$ & $2,077(18)$ & \\
\hline II & $42,547(16)$ & $41,013(16)$ & $1,534(13)$ & \\
\hline III & $47,198(17)$ & $45,381(17)$ & $1,817(16)$ & \\
\hline IV & $56,547(21)$ & $54,269(21)$ & $2,278(20)$ & \\
\hline $\mathrm{V}$ & $80,736(29)$ & $77,027(29)$ & $2,709(33)$ & \\
\hline Hypertension & $107,627(39)$ & $98,455(37)$ & $9,172(80)$ & $<0.001$ \\
\hline Diabetes & $41,999(15)$ & $36,374(14)$ & $5,625(49)$ & $<0.001$ \\
\hline Dyslipidemia & $60,682(22)$ & $49,461(19)$ & $11,221(98)$ & $<0.001$ \\
\hline Ischemic heart disease (I2O) & $9,587(4)$ & $7,267(3)$ & $2,320(20)$ & $<0.001$ \\
\hline Transient ischemic attack & $2,270(1)$ & $1,946(1)$ & $324(3)$ & $<0.001$ \\
\hline Heart failure & $4,894(2)$ & $4,081(2)$ & $813(7)$ & $<0.001$ \\
\hline Atrial fibrillation & $1,474(1)$ & $1,250(1)$ & $224(2)$ & $<0.001$ \\
\hline Valvular heart disease & $800(0)$ & $678(0)$ & $122(1)$ & $<0.001$ \\
\hline Chronic renal disease & $2,144(1)$ & $1,760(1)$ & $384(3)$ & $<0.001$ \\
\hline
\end{tabular}


Table 1. Continued

\begin{tabular}{|c|c|c|c|c|}
\hline Characteristic & $\begin{array}{c}\text { Total } \\
(\mathrm{n}=275,382)\end{array}$ & $\begin{array}{l}\text { Non-statin users } \\
\quad(n=263,967)\end{array}$ & $\begin{array}{l}\text { Statin users } \\
(\mathrm{n}=11,415)\end{array}$ & $p$ value \\
\hline Carotid stenosis & $157(0)$ & $74(0)$ & $83(1)$ & $<0.001$ \\
\hline Peripheral vascular disease & $12,625(5)$ & $10,406(4)$ & $2,219(19)$ & $<0.001$ \\
\hline Chronic liver disease & $2,221(1)$ & $2,086(1)$ & $135(1)$ & $<0.001$ \\
\hline Rheumatoid disease & $10,021(4)$ & $9,325(4)$ & $696(6)$ & $<0.001$ \\
\hline Inflammatory bowel disease & $783(0)$ & $754(0)$ & $29(0)$ & 0.535 \\
\hline $\begin{array}{l}\text { Malignancy including solid cancer and } \\
\text { hematologic malignancy }\end{array}$ & $8,333(3)$ & $7,803(3)$ & $530(5)$ & $<0.001$ \\
\hline $\begin{array}{l}\text { Recipients of solid organ transplantation } \\
\text { or hematopoietic stem cell transplantation }\end{array}$ & $90(0)$ & $84(0)$ & $6(0)$ & 0.279 \\
\hline Human immunodeficiency virus infection & $16(0)$ & $15(0)$ & $1(0)$ & 0.492 \\
\hline Depression & $1,985(1)$ & $1,819(1)$ & $166(2)$ & $<0.001$ \\
\hline Herpes zoster ${ }^{\mathrm{a}}$ & $15,439(6)$ & $13,516(5)$ & $1,923(17)$ & $<0.001$ \\
\hline
\end{tabular}

Values are presented as mean \pm SD or number (\%).

BMI, body mass index.

${ }^{\text {aT } T h i s ~ v a r i a b l e ~ i s ~ n o t ~ a ~ b a s e l i n e ~ v a r i a b l e ~ b e t w e e n ~ n o n-s t a t i n ~ u s e r s ~ a n d ~ s t a t i n ~ u s e r s . ~ T h i s ~ v a r i a b l e ~ i s ~ t h e ~ d e v e l o p m e n t ~ o f ~ h e r p e s ~}$ zoster during the follow-up period over 12 years.

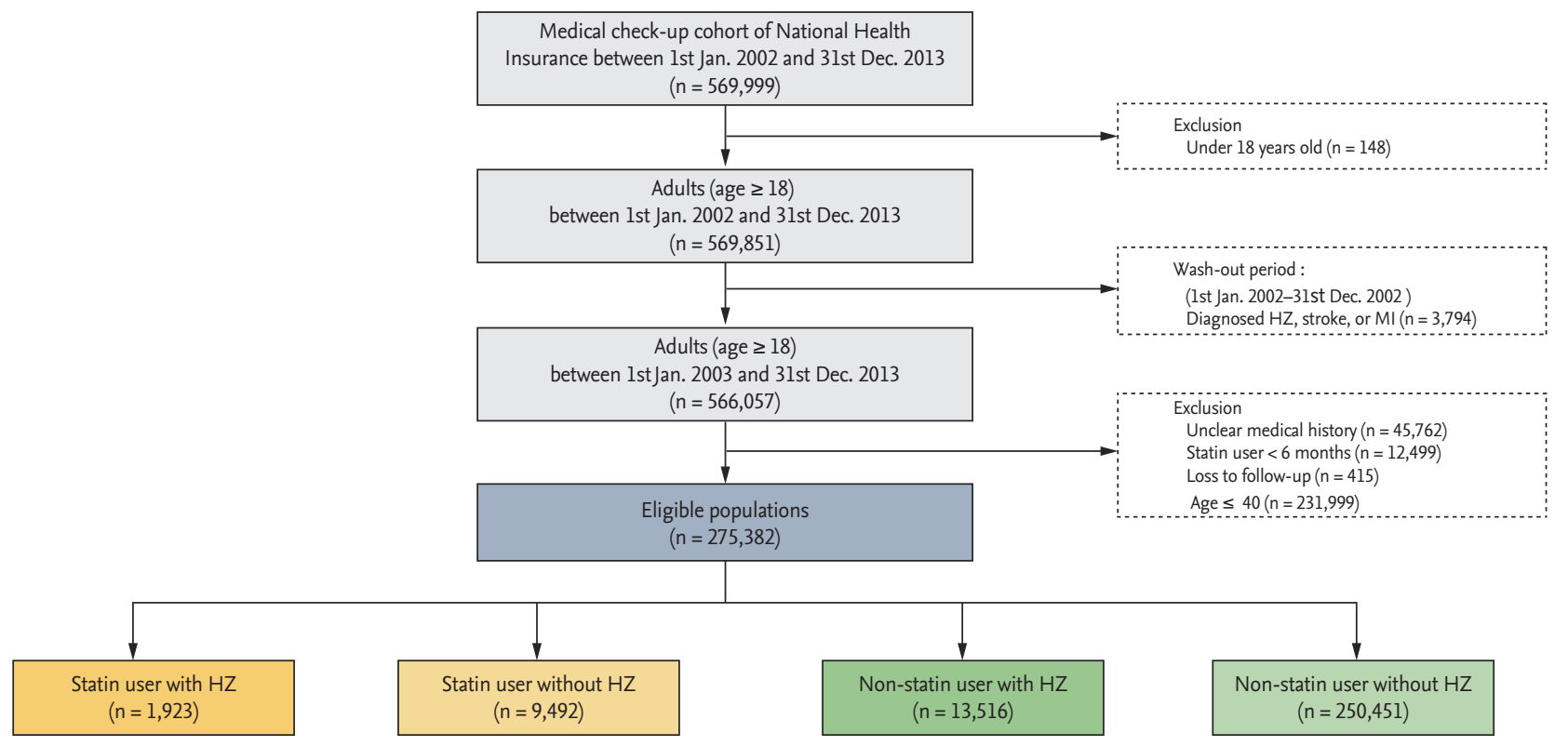

Figure 1. Flow diagram of the study. HZ, herpes zoster; MI, myocardial infarction.

years old were excluded, and a total 569,851 persons $\geq$ 18 years old were followed up for 11 years from 2002 through 2013. To identify the first-ever episodes of HZ, stroke, and MI, we excluded individuals with evidence of these diseases during the first year of the observation period, which we designated as a wash-out period. As a result, we excluded 3,794 patients with a history of HZ, stroke, and MI. Furthermore, patients who lacked medical records prior to the occurrence of HZ, stroke, and MI were excluded $(n=45,762)$. All patients were required to have used statins for at least 6 months prior to the beginning of the study period; those who used statins for 


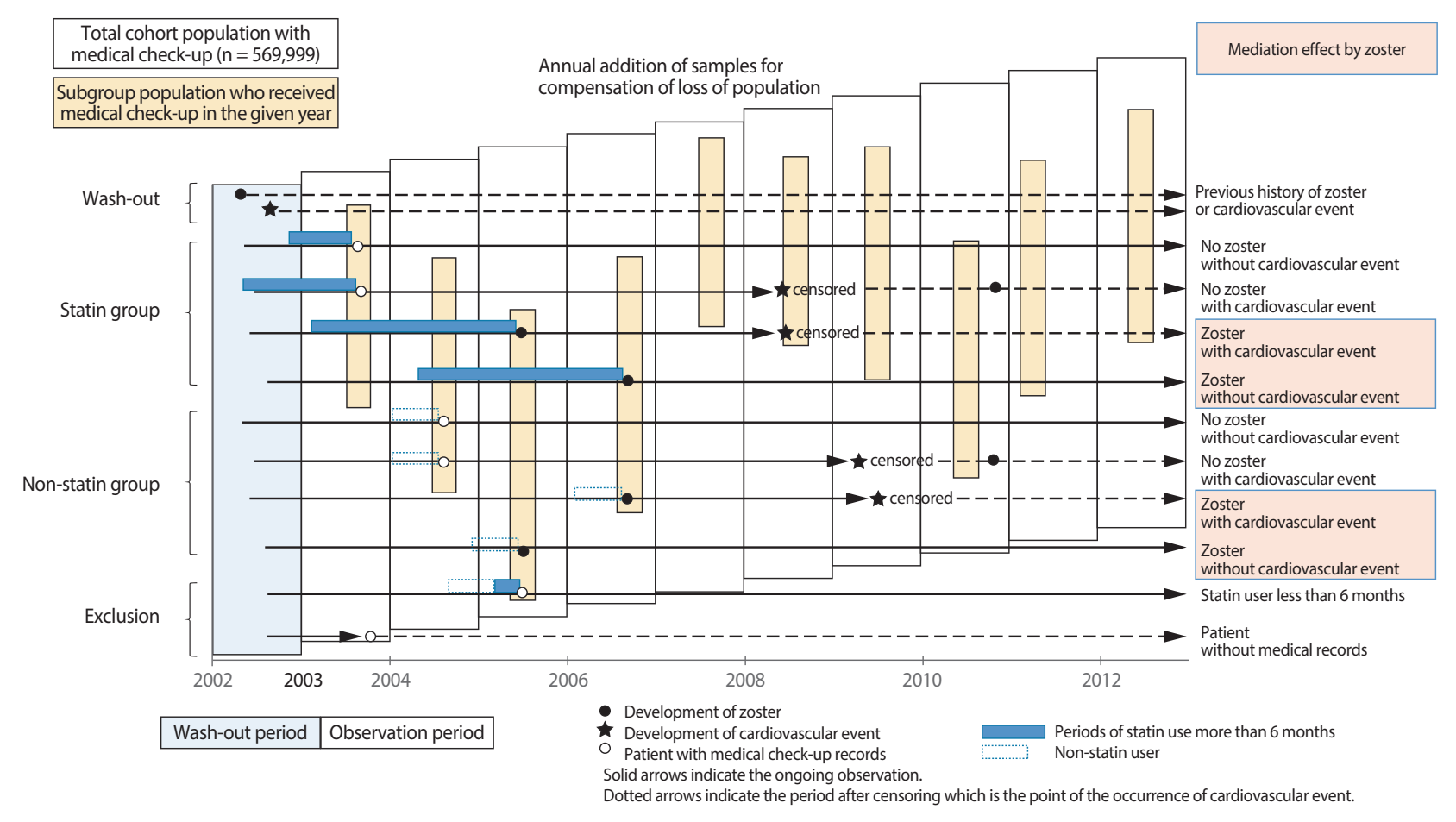

Figure 2. Schematic diagram of the study.

less than 6 months prior to the beginning of the study period were excluded $(n=12,499)$. Individuals lost to follow up were also excluded $(\mathrm{n}=415)$. In addition, people $\leq$ 40 years old were excluded due to low prescription rate of statin. A total of 275,382 persons were finally observed over an 11-year period from 2003 to 2013 (Fig. 1). A detailed schematic diagram is shown in Fig. 2. Furthermore, we performed subgroup analyses according to subtypes of age groups, considering the different risks of stroke after HZ by age groups [2].

\section{Indirect or mediation effect}

Our main interest was quantifying whether or not, and by how much, the effect of the statins on CVD is mediated by HZ. The total effect of statins on CVD is decomposed by natural direct and indirect effects (or mediation effect) operating through mediator HZ (Supplementary Fig. 1). The definitions of natural direct and indirect effects within a potential outcome framework have been published elsewhere [12].

\section{Statistical analysis}

Categorical variables are presented as numbers and percentages, and were compared using the Pearson's chi- square test. Continuous variables are expressed as mean $\pm \mathrm{SD}$, and were compared using the Student's $t$ test.

We performed the two-stage regression methods proposed by VanderWeele to evaluate the natural direct and indirect effects [12]. First, we fit a logistic regression for mediator HZ conditional on statin use and confounders. We then fit a Cox proportional hazards regression model for CVD risk on statin, HZ, statin-HZ interaction term, and confounders. Because the statin-HZ interaction effect was not statistically significant, we dropped the exposure-mediator interaction from the final model. The natural direct and indirect effects were estimated using the coefficients of the above regressions and expression provided in VanderWeele's book [11]. Finally, we calculated the "percentage of proportion mediated"

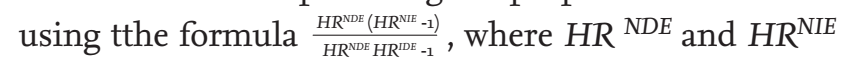
are the natural direct and indirect effects of the hazard ratio, respectively. This measure evaluates how much of the overall effect of statins on CVD was due to the effect of statins on the mediator. In the sensitivity analysis, we included the variable chronic pulmonary disease as a variable in order to quantify the impact of unmeasured mediator-outcome confounding on the mediation effect, because chronic pulmonary disease is a well-known 
Table 2. Statin use, subsequent herpes zoster, and the mediating effect of cardiovascular disease by statin use through herpes zoster $^{\text {a }}$

\begin{tabular}{|c|c|c|c|c|c|c|c|c|c|}
\hline \multirow{2}{*}{ Variable } & \multicolumn{3}{|c|}{ MI } & \multicolumn{3}{|c|}{ Stroke } & \multicolumn{3}{|c|}{ Cardiovascular disease } \\
\hline & aHR & $95 \% \mathrm{CI}$ & $p$ value & aHR & $95 \% \mathrm{CI}$ & $p$ value & aHR & $95 \% \mathrm{CI}$ & $p$ value \\
\hline \multicolumn{10}{|l|}{ Statin use } \\
\hline $\mathrm{NDE}$ & 0.899 & $0.772-1.048$ & 0.1743 & 0.873 & $0.791-0.964$ & 0.0072 & 0.885 & $0.812-0.964$ & 0.0051 \\
\hline $\mathrm{ME} / \mathrm{NIE}$ & 1.016 & $1.004-1.029$ & 0.0082 & 1.011 & $1.004-1.018$ & 0.0032 & 1.014 & $1.006-1.020$ & $<0.0001$ \\
\hline Total effect & 0.914 & $0.785-1.065$ & 0.2502 & 0.883 & $0.800-0.975$ & 0.0135 & 0.897 & $0.823-0.977$ & 0.0125 \\
\hline Proportion mediated, \% & & -17.28 & & & -8.22 & & & -11.56 & \\
\hline Herpes zoster & 1.274 & $1.087-1.493$ & 0.0028 & 1.182 & $1.068-1.308$ & 0.0012 & 1.224 & $1.121-1.336$ & $<0.0001$ \\
\hline Interaction & & & 0.9525 & & & 0.9379 & & & 0.5616 \\
\hline \multicolumn{10}{|l|}{ Subgroup analysis } \\
\hline \multicolumn{10}{|l|}{$<60$ years } \\
\hline $\mathrm{NDE}$ & 0.836 & $0.643-1.088$ & 0.1826 & 1.013 & $0.849-1.210$ & 0.8835 & 0.944 & $0.812-1.098$ & 0.4575 \\
\hline $\mathrm{ME} / \mathrm{NIE}$ & 1.036 & $1.012-1.060$ & 0.0029 & 1.019 & $1.004-1.034$ & 0.0116 & 1.026 & $1.013-1.040$ & $<0.0001$ \\
\hline Total effect & 0.866 & $0.656-1.126$ & 0.2836 & 1.033 & $0.865-1.232$ & 0.723 & 0.969 & $0.834-1.127$ & 0.6855 \\
\hline \multicolumn{10}{|l|}{$\geq 60$ years } \\
\hline $\mathrm{NDE}$ & 0.969 & $0.802-1.171$ & 0.7419 & 0.838 & $0.744-0.944$ & 0.0035 & 0.882 & $0.795-0.979$ & 0.0188 \\
\hline $\mathrm{ME} / \mathrm{NIE}$ & 1.006 & $0.993-1.019$ & 0.3416 & 1.007 & $1.000-1.015$ & 0.064 & 1.007 & $1.000-1.014$ & 0.0387 \\
\hline Total effect & 0.975 & $0.807-1.178$ & 0.7904 & 0.844 & $0.749-0.950$ & 0.0051 & 0.889 & $0.800-0.986$ & 0.0267 \\
\hline
\end{tabular}

MI, myocardial infarction; aHR, adjusted hazard ratios; CI, confidence interval; NDE, natural direct effect; ME, mediation effect; NIE, natural indirect effect.

${ }^{a}$ Adjusted for age, male gender, hypertension, hyperlipidemia, ischemic heart disease, diabetes, heart failure, peripheral vascular disease, arterial fibrillation or atrial flutter, renal disease, and valvular heart disease.

major risk factor for $\mathrm{HZ}$ and CVD [11]. Also, we conducted additional sensitivity analyses to examine the robustness of our results. All reported $p$ values are two-sided, and $p$ values of $<0.05$ were considered statistically significant. Data manipulation and statistical analyses were conducted using SAS version 9.2 (SAS Institute Inc., Cary, NC, USA) and a macro of $\%$ mediation [13].

\section{RESULTS}

\section{Study population}

Table 1 presents the baseline clinical characteristics in the cohort. Of a total of 275,382 study subjects, 13,849 individuals (4.9\%) developed cardiovascular events during the 12-year follow-up while 15,439 (5.6\%) subjects developed herpes zoster. Of total study subjects, 11,415 (4.1\%) individuals were using statin.
The multivariate analysis on the risk factors for stroke, MI, CVD, and zoster are shown in Supplementary Table 1. Old age, high body mass index, obesity, diabetes, dyslipidemia, angina pectoris, peripheral vascular disease, and rheumatoid disease were positively associated with CVD and zoster. While, male gender was more common in patients with CVD, while female gender was more common in HZ patients. However, the HZ patients were more likely to be higher income groups and non-smokers, while CVD patients were less likely to be high income groups and smokers.

\section{Statin use, the occurrence of $\mathrm{HZ}$, and the CVD}

The occurrence of zoster was significantly associated with statin use (adjusted odds ratio [OR], 2.47; 95\% confidence interval [CI], 2.32 to 2.64) (Supplementary Table 1). The total effects of the stroke, MI, and CVD in the statin group were lower than in the non-statin group (Table 
2); the adjusted hazard ratios (HRs) were 0.90 (95\% CI, 0.82 to 0.98 ), 0.88 ( $95 \%$ CI, 0.80 to 0.98 ), and 0.91 ( $95 \% \mathrm{CI}$, 0.79 to 1.07 ), respectively. However, the CVD was higher in patients who experienced HZ than in those who did not (Table 2); the adjusted HRs were 1.22 (95\% CI, 1.12 to 1.34 ), 1.18 (95\% CI, 1.07 to 1.31), and 1.27 (95\% CI, 1.09 to 1.49), respectively. We observed significant indirect or mediation effects of statin use on the CVD; the adjusted HRs were 1.01 (95\% CI, 1.01 to 1.02), 1.01 (95\% CI, 1.00 to 1.02 ), and 1.02 (95\% CI, 1.00 to 1.03 ), respectively. The direct effects of adjusted HRs for statin use, through pathways other than $\mathrm{HZ}$, were 0.89 ( $95 \% \mathrm{CI}, 0.81$ to 0.96 ), 0.87 (95\% CI, 0.79 to 0.96 ), and 0.90 (95\% CI, 0.77 to 1.05 ) for the risk of the stroke, MI, and CVD, respectively. The direct effect of statin use was, through pathways other than HZ, a bit bigger than the total effect since statin use increased the incidence of HZ and thereby also increased CVD. When we calculated the mediating effect of cardiovascular events by statin use through HZ, $11.6 \%$ of the total beneficial effect of statin use on CVD was mitigated through the occurrence of statin-induced HZ. In addition, this mediating effect was higher in the younger age group ( $<60$ years) than in older age group ( $\geq$ 60 years); the adjusted HRs of the mediation effects were 1.03 ( $95 \%$ CI, 1.01 to 1.04) and 1.01 (95\% CI, 1.00 to 1.01) for the risk of the CVD in the younger age group and older age group, respectively (Table 2).

\section{Sensitivity analysis}

First, we tried to validate our findings by using "chronic pulmonary disease," which was not included in the multivariable analysis, as one of the unmeasured confounding variables for the sensitivity analysis. The number of patients with chronic pulmonary disease was 3,888 (8.4\%) for the HZ group and 42,361 (16.3\%) for the NonHZ group. The adjusted OR for HZ was 1.52 (95\% CI, 1.47 to 1.59), and the adjusted HRs were 1.12 (95\% CI, 1.07 to 1.16 ), 1.07 (95\% CI, 1.02 to 1.12), and 1.24 (95\% CI, 1.15 to 1.34) for the risk of the stroke, MI, and CVD, respectively; the mediation effect through the occurrence of HZ caused by statin use to prevent cardiovascular events was 10.8\%. Second, we included individuals between 18 and 40 years old who were excluded in the main analysis due to a low prescription rate of statin for sensitivity analysis. In this scenario, the mediation effect through the occurrence of HZ caused by statin use to prevent car- diovascular events was 9.5\%. Third, we included people between the ages of 18 and 40 years who used statins for less than 6 months prior to the study initiation as part of the non-statin group. The mediation effect through the occurrence of HZ caused by statin use to prevent cardiovascular events in this scenario was 9.1\%. The detailed results of these sensitivity analyses are presented in Supplementary Table 2.

\section{DISCUSSION}

We have demonstrated the effects of statin use on the CVD through (1) being associated with decreasing the risk of stroke and MI while (2) increasing the risk of $\mathrm{HZ}$, which in turn was associated with increasing the risk of stroke and MI. In addition, we showed that the protective effect of statins on stroke and MI was mitigated up to around $10 \%$ due to the increased incidence of HZ (about one thousand of ten thousand statin users). Therefore, our data suggest that $\mathrm{HZ}$ vaccination might potentiate the beneficial effect of statin use by preventing the development of $\mathrm{HZ}$, although we did not directly evaluate the role of zoster vaccination on cardiovascular events among statin users. Given that physicians prescribe statins to patients with the same risk factors for the development of HZ, our measurement of this mediating effect of HZ caused by statin use is important in order to make a policy decision for the necessity of zoster vaccination in patients who receive statins.

It is well-known that statins reduce the risk of CVD-associated morbidity and mortality through their effects on lipids and are also thought to have anti-inflammatory and other plaque-stabilization effects [14]. However, recent studies have consistently reported a small but significantly increased risk of HZ in patients receiving statin treatment, and that this risk increases in a dose-dependent manner [7-9]. Several possible mechanisms have been proposed, including the increase of regulatory $\mathrm{T}$ cells and impaired $\mathrm{Th} 1$ responses [6-9]. Given that previous epidemiologic studies have reported similar increased risks of cardiovascular events following HZ [1-5], it can be easily extrapolated that the protective effects of statins on CVD may be mitigated by a small but significant increased risk of HZ caused by statin use, subsequently increasing the risk of CVD. 
Furthermore, since a recent zoster vaccine trial reported more than $95 \%$ vaccine efficacy [15], the mediating effect of CVD by statin use through HZ may be counterbalanced by zoster vaccination. Considering the approximate $10 \%$ mediating effect of statin use through HZ, clinicians should be mindful of this epidemiologic association until further epidemiologic studies have elucidated the counter-mediating effect of zoster vaccination on cardiovascular outcomes in patients who receive statin treatment. It is also crucial to inform patients that the protective effects of statins against CVD may be mitigated by the increased risk of HZ from taking statins, and that zoster vaccination might be beneficial in these patients.

It is noteworthy that the mediating effect of CVD by statin use through HZ was higher in younger patients who received statin treatment (Table 2). A previous study revealed that the risk of stroke after HZ was highest in the younger age group and gradually diminished with age [2]. Therefore, HZ is an important contributing factor to the development of CVD in a relatively younger population with fewer risk factors for atherosclerosis. Taking this into consideration, individuals under 60 years old who receive statin treatment to prevent CVD might gain a more beneficial effect from zoster vaccination. In South Korea, the prevalence of statin use is not high due to the relative low prevalence of hyperlipidemia (4.1\% in this study). However, national data based on the Korea National Health and Nutrition Examination Survey show an increasing trend of hyperlimidemia prevalence especially among young adult South Koreans [16], which in turn might well result in wider use of statin among young adults. In this context, the necessity of zoster vaccination in individuals under 60 years old should be discussed, and further clinical trials are needed to determine the safety and efficacy of the zoster vaccine in this population.

This study has several limitations. First, some may be concerned that this study included subjects who received medical check-ups, thus making this a biased cohort population. However, the agreement between the incidences of specific clinical characteristics in this study, such as hypertension and diabetes (Table $1)$, and the real prevalence of these diseases in South Korea [16] supports the representative nature of our cohort. Second, the validity of HZ diagnosis based on
ICD codes might be questioned. However, the diagnostic codes have been validated and shown to have high sensitivity and positive predictive values (> 85\%) for the identification of new cases of HZ [17,18], Furthermore, any misclassification caused by the ICD diagnostic codes would more likely lead to a bias towards the null. Third, similar to other observational studies, the validity of our results depend on the assumption of there being no unmeasured confounding variables [12]. Our limited sensitivity analysis results were not substantially different from the main results, but require careful interpretation.

In conclusion, this study suggested that HZ vaccination among statin users may partly reduce the risk of CVD. Further studies including randomized clinical trials are warranted to determine the causal effect of zoster vaccination among statin users on CVD.

\section{KEY MESSAGE}

1. Previous epidemiologic studies revealed that herpes zoster (HZ) increases the risk of cardiovascular events including stroke and myocardial infarction (MI). It is also well-known that statin use reduces the risk of stroke and MI. However, recent studies have consistently reported that statin use increases the incidence of $\mathrm{HZ}$.

2. We assessed the mediation effect of HZ caused by statin use to prevent stroke and MI. We showed that the protective effect of statin on stroke and MI was mitigated up to around 10\% due to the increased incidence of HZ (around thousands of 10 thousand statin users). This mediating effect was higher in the younger age group ( $<60$ years).

3. Our data suggest that zoster vaccination in patients who receive statins may potentiate the beneficial effect of statin use through preventing the development of $\mathrm{HZ}$ after statin.

\section{Conflict of interest}

No potential conflict of interest relevant to this article was reported. 


\section{Acknowledgments}

This study was supported by a grant from the Korea Health Technology R\&D Project through the Korea Health Industry Development Institute (KHIDI) and the National Research Foundation of Korea (NRF) (No. 2018R1D1A1A09083902), funded by the Ministry of Health and Welfare, Republic of Korea (grant no: $\mathrm{HI}_{15} \mathrm{C}_{1763)}$.

\section{REFERENCES}

1. Kwon SU, Yun SC, Kim MC, et al. Risk of stroke and transient ischaemic attack after herpes zoster. Clin Microbiol Infect 2016;22:542-548.

2. Kim MC, Yun SC, Lee HB, et al. Herpes zoster increases the risk of stroke and myocardial infarction. J Am Coll Cardiol 2017;70:295-296.

3. Breuer J, Pacou M, Gauthier A, Brown MM. Herpes zoster as a risk factor for stroke and TIA: a retrospective cohort study in the UK. Neurology 2014;82:206-212.

4. Langan SM, Minassian C, Smeeth L, Thomas SL. Risk of stroke following herpes zoster: a self-controlled case-series study. Clin Infect Dis 2014;58:1497-1503.

5. Yawn BP, Wollan PC, Nagel MA, Gilden D. Risk of stroke and myocardial infarction after herpes zoster in older adults in a US community population. Mayo Clin Proc 2016;91:33-44.

6. Chou R, Dana T, Blazina I, Daeges M, Jeanne TL. Statins for prevention of cardiovascular disease in adults: evidence report and systematic review for the US Preventive services task force. JAMA 2016;316:2008-2024.

7. Antoniou T, Zheng H, Singh S, Juurlink DN, Mamdani MM, Gomes T. Statins and the risk of herpes zoster: a population-based cohort study. Clin Infect Dis 2014;58:350-356.

8. Chen HH, Lin CL, Yeh CJ, Yeh SY, Kao CH. Statins can increase the risk of herpes zoster infection in Asia. Eur J
Clin Microbiol Infect Dis 2015;34:1451-1458.

9. Matthews A, Turkson M, Forbes H, Langan SM, Smeeth

L, Bhaskaran K. Statin use and the risk of herpes zoster: a nested case-control study using primary care data from the U.K. Clinical Research Practice Datalink. Br J Dermatol 2016;175:1183-1194.

10. Lee J, Lee JS, Park SH, Shin SA, Kim K. Cohort profile: the National Health Insurance Service-National Sample Cohort (NHIS-NSC), South Korea. Int J Epidemiol 2017;46:e15.

11. VanderWeele TJ. Explanation in Causal Inference: Methods for Mediation and Interaction. New York (NY): Oxford University Press, 2015.

12. Yang YW, Chen YH, Wang KH, Wang CY, Lin HW. Risk of herpes zoster among patients with chronic obstructive pulmonary disease: a population-based study. CMAJ 2011;183:E275-E280.

13. Valeri L, Vanderweele TJ. Mediation analysis allowing for exposure-mediator interactions and causal interpretation: theoretical assumptions and implementation with SAS and SPSS macros. Psychol Methods 2013;18:137-150.

14. Charo IF, Taub R. Anti-inflammatory therapeutics for the treatment of atherosclerosis. Nat Rev Drug Discov 2011;10:365-376.

15. Lal H, Cunningham AL, Godeaux O, et al. Efficacy of an adjuvanted herpes zoster subunit vaccine in older adults. N Engl J Med 2015;372:2087-2096.

16. Korea Center for Disease Control and Prevention. Korea National Health and Nutrition Examination Survey 2013 [Internet]. Cheongju (KR): KCDC, 2019 [cited 2019 Nov 2o]. Available from: https://knhanes.cdc.go.kr/knhanes/ index.do.

17. Donahue JG, Choo PW, Manson JE, Platt R. The incidence of herpes zoster. Arch Intern Med 1995;155:1605-1609.

18. Mullooly JP, Riedlinger K, Chun C, Weinmann S, Houston H. Incidence of herpes zoster, 1997-2002. Epidemiol Infect 2005;133:245-253. 


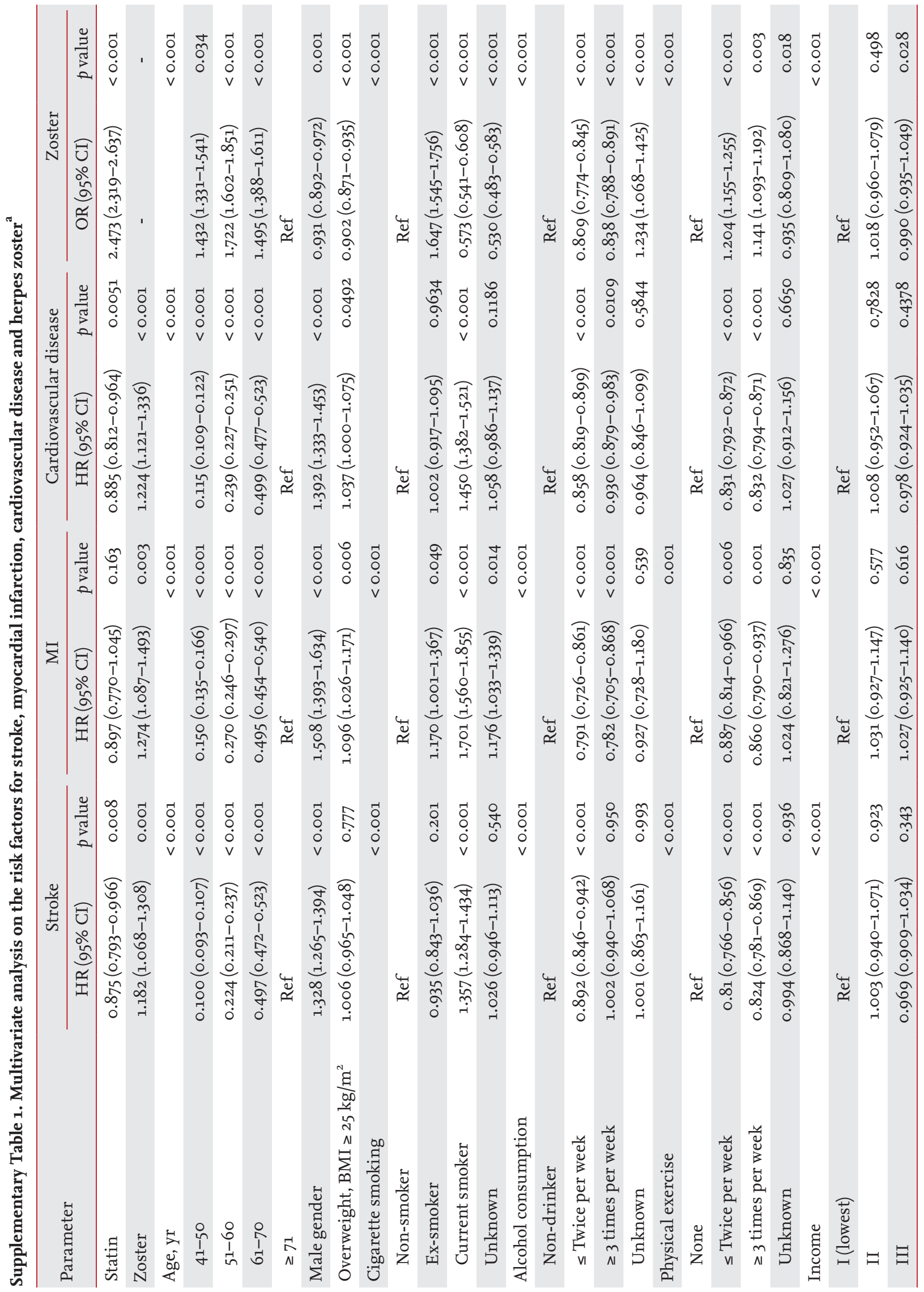




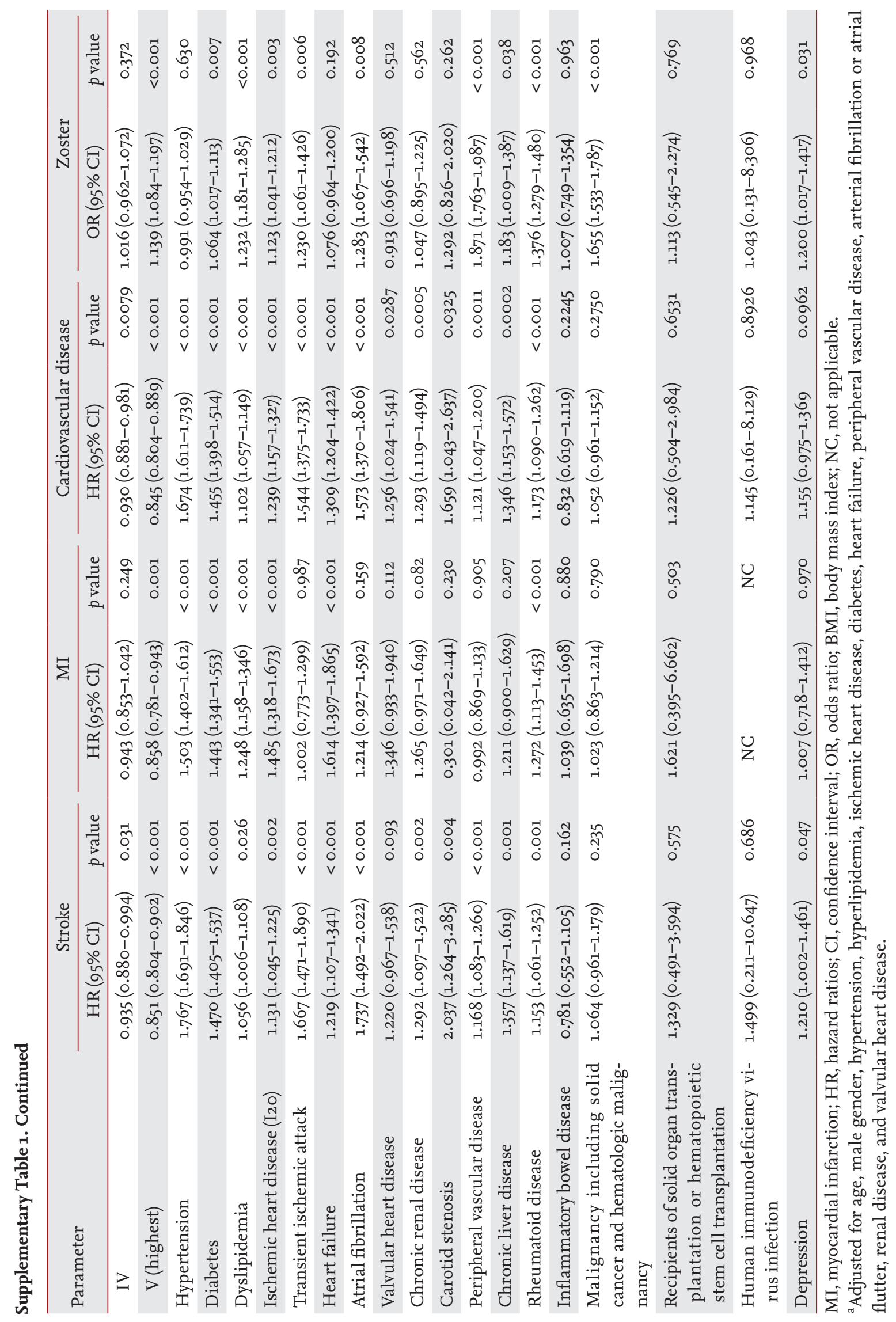


Supplementary Table 2. Multiple sensitivity analysis for statin use, subsequent herpes zoster, and the mediating effect of cardiovascular disease by statin use through herpes zoster ${ }^{a}$

\begin{tabular}{|c|c|c|c|c|c|c|c|c|c|}
\hline \multirow{2}{*}{ Variable } & \multicolumn{3}{|c|}{ MI } & \multicolumn{3}{|c|}{ Stroke } & \multicolumn{3}{|c|}{ Cardiovascular disease } \\
\hline & aHR & $95 \% \mathrm{CI}$ & $p$ value & aHR & $95 \% \mathrm{CI}$ & $p$ value & aHR & $95 \% \mathrm{CI}$ & $p$ value \\
\hline \multicolumn{10}{|c|}{ Chronic pulmonary disease included in multivariate analysis } \\
\hline NDE & 0.900 & $0.772-1.048$ & 0.1760 & 0.873 & $0.791-0.964$ & 0.0071 & 0.884 & $0.812-0.964$ & 0.0050 \\
\hline $\mathrm{ME} / \mathrm{NIE}$ & 1.015 & $1.003-1.027$ & 0.0118 & 1.010 & $1.003-1.018$ & 0.0039 & 1.013 & $1.006-1.019$ & $<0.0001$ \\
\hline Total effect & 0.9134 & $0.784-1.064$ & 0.2454 & 0.883 & $0.800-0.974$ & 0.0130 & 0.896 & $0.822-0.976$ & 0.0118 \\
\hline Proportion mediated, \% & & $-15 \cdot 72$ & & & -7.78 & & & -10.79 & \\
\hline \multicolumn{10}{|c|}{ People between 18 and 40 years included } \\
\hline $\mathrm{NDE}$ & 0.905 & $0.779-1.051$ & 0.1897 & 0.869 & $0.788-0.958$ & 0.0049 & 0.883 & $0.811-0.961$ & 0.0039 \\
\hline $\mathrm{ME} / \mathrm{NIE}$ & 1.013 & $1.004-1.023$ & 0.0050 & 1.010 & $1.004-1.016$ & 0.0007 & 1.012 & $1.006-1.017$ & $<0.0001$ \\
\hline Total effect & 0.917 & $0.789-1.065$ & 0.2547 & 0.877 & $0.796-0.968$ & 0.0084 & 0.893 & $0.820-0.972$ & 0.0087 \\
\hline Proportion mediated, \% & & -14.50 & & & -6.98 & & & $-9 \cdot 52$ & \\
\hline \multicolumn{10}{|c|}{ People between 18 and 40 years old who used statin for less than 6 months prior to study initiation included as non-statin group } \\
\hline NDE & 0.890 & $0.769-1.031$ & 0.1197 & 0.885 & $0.804-0.975$ & 0.0129 & 0.891 & $0.820-0.968$ & 0.0065 \\
\hline $\mathrm{ME} / \mathrm{NIE}$ & 1.012 & $1.003-1.021$ & 0.0072 & 1.009 & $1.003-1.014$ & 0.0016 & 1.010 & $1.006-1.015$ & $<0.0001$ \\
\hline Total effect & 0.901 & $0.778-1.043$ & 0.1617 & 0.893 & $0.811-0.983$ & 0.0206 & 0.900 & $0.828-0.978$ & 0.0130 \\
\hline Proportion mediated, \% & & -10.67 & & & -7.05 & & & -9.14 & \\
\hline
\end{tabular}

MI, myocardial infarction; aHR, adjusted hazard ratios; CI, confidence interval; NDE, natural direct effect; ME, mediation effect; NIE, natural indirect effect.

${ }^{a}$ Adjusted for age, male gender, hypertension, hyperlipidemia, ischemic heart disease, diabetes, heart failure, peripheral vascular disease, arterial fibrillation or atrial flutter, renal disease, and valvular heart disease. 


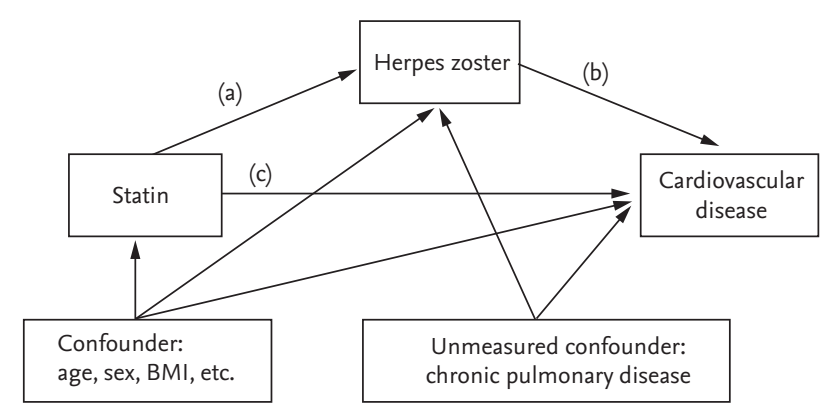

Supplementary Figure 1. The association of statin, herpes zoster, and cardiovascular disease with measured and unmeasured confounding variables. BMI, body mass index. 